\title{
An improved technique for culture of catheter tips from open-heart surgery patients
}

\author{
R FREEMAN, N HJERSING
}

From the Department of Microbiology, Freeman Hospital, Newcastle upon Tyne

ABSTRACT Bacteria were isolated from $19.6 \%$ of left atrial catheter tips from open-heart surgical patients when incubation of the culture was prolonged to eight days and the prophylactic antibiotic given to the patient (flucloxacillin) was specifically neutralised with beta-lactamase. This compares with the previous estimate of $9.9 \%$ when a standard culture method was employed. Additionally, diphtheroid organisms were recovered from left atrial catheter tips using this new technique.

The recovery of organisms similar to those involved in early prosthetic valve endocarditis (early PVE) from the tips of left atrial catheters in patients undergoing open-heart surgery has led to the suggestion that these catheter tips might form one of the reservoirs of organisms from which early PVE is derived. A previous report from this unit set the isolation rate of such organisms from left atrial catheter tips at $9.9 \%{ }^{1}$ However, this figure was derived from a system in which the prophylactic antibiotic being given to each patient (flucloxacillin) was not neutralised. In view of the previous work on the culture of perfusion blood, in which it was shown that the addition of a suitable beta-lactamase enzyme to the culture system resulted in markedly increased yield of positive cultures, ${ }^{2}$ it was decided to look again at catheter tip cultures to assess whether un-neutralised prophylactic antibiotic was significantly masking the isolation rate.

Further, although the organisms previously isolated from left atrial catheter tips were typical of those found in PVE, that is they were coagulase negative staphylococci of various types, it was observed that the other common group of organisms found in early PVE-diphtheroids - were rarely isolated from this site. Since diphtheroids are slowly growing bacteria, it was also thought necessary to investigate the effects of prolonging the time of incubation of catheter-tip cultures. In this report we describe the results of applying these two variations to the routine technique on the isolation rates of organisms from left atrial catheter tips.

\section{Methods}

One hundred and sixty-one left atrial catheter tips were obtained and cultured as previously described. ${ }^{3}$ It is important to note that the duration of incubation

Address for reprint requests: Dr R Freeman, Department of Microbiology, Freeman Hospital, Freeman Road, High Heaton, Newcastle upon Tyne NE7 7DN. was 48 hours. These specimens are subsequently referred to as "standard method" specimens, and formed part of a series reported earlier. ${ }^{1} \mathrm{~A}$ further 201 consecutive left atrial catheter tips were then examined in a modified manner. The modifications were carried out in two stages:

1 After receipt in the laboratory, sterile broth was added with aseptic precautions to the severed catheter tip in a Universal container, the resultant specimen being then incubated at $37^{\circ} \mathrm{C}$. The broth was inspected for turbidity daily, culture being performed only if signs of bacterial growth appeared, or, in any event, after eight days of incubation. No beta-lactamase was added to these cultures. This modification was applied to 33 consecutive catheter tips.

2 The remaining 168 catheter tips were cultured as described above, with the single extra step of the addition of an excess of type II beta-lactamase at the start of the procedure. All patients from whom these specimens were obtained were receiving flucloxacillin as the sole antibiotic prophylaxis at the time of catheter removal.

All organisms isolated were identified by standard methods.

\section{Results}

Table 1 reveals that the yield from a system including both prolonged incubation and the addition of beta-lactamase is significantly increased compared with the standard method $(\mathrm{p}=<0.05)$. Although the effect of prolonged incubation alone was assessed on a relatively small number of specimens, it does not appear to be as effective as the combination of both prolonged incubation and beta-lactamase addition. However, the number of specimens in this category is too small to allow accurate comparison.

In table 2 it is seen that the distribution of bacterial isolates per day of incubation in the system 
Table 1 Effects of prolonging the incubation time and the addition of beta-lactamase on the isolation rate of organisms from left atrial catheter tips

\begin{tabular}{|c|c|c|c|c|c|c|}
\hline \multirow[t]{2}{*}{ Method } & \multirow{2}{*}{$\begin{array}{l}\text { Number of } \\
\text { catheters }\end{array}$} & \multirow{2}{*}{$\begin{array}{l}\text { Number } \\
\text { positive }\end{array}$} & \multicolumn{4}{|c|}{ Varieties of organisms isolated } \\
\hline & & & CNS & $D I P H$ & $S T R E P \ddagger$ & Other \\
\hline $\begin{array}{l}\text { Standard } \\
\text { Long incubation alone } \\
\text { Long incubation }+ \text { beta-lactamase }\end{array}$ & $\begin{array}{r}161 \\
33 \\
168\end{array}$ & $\begin{array}{c}16(9 \cdot 9 \%) \\
3(9 \cdot 1 \%) \\
33(19.6 \%)\end{array}$ & $\begin{array}{l}13(8 \%) \\
2(6 \%) \\
22(13 \cdot 1 \%)\end{array}$ & $\begin{array}{l}1(0.6 \%) \\
1(3 \%) \\
6(3.6 \%)\end{array}$ & $\begin{array}{l}1(0 \cdot 6 \%) \\
0 \\
3(1.8 \%)\end{array}$ & $\begin{array}{l}1(0.6 \%)^{*} \\
0 \\
2(1.2 \%) \dagger\end{array}$ \\
\hline
\end{tabular}

*This organism was Escherichia coli.

$\dagger$ These organisms were a Flavobacterium and a Micrococcus.

Key: CNS = coagulase negative staphylococci; DIPH = diphtheroids; STREP = streptococci.

$¥$ All the streptococci isolated were of the Strep viridans type.

Statistical note: when the "standard" method is compared with the "Long incubation + beta-lactamase" technique $p=<0 \cdot 05$.

Table 2 Relationship between the duration of incubation and the isolation of the various organisms from left atrial catheter tips

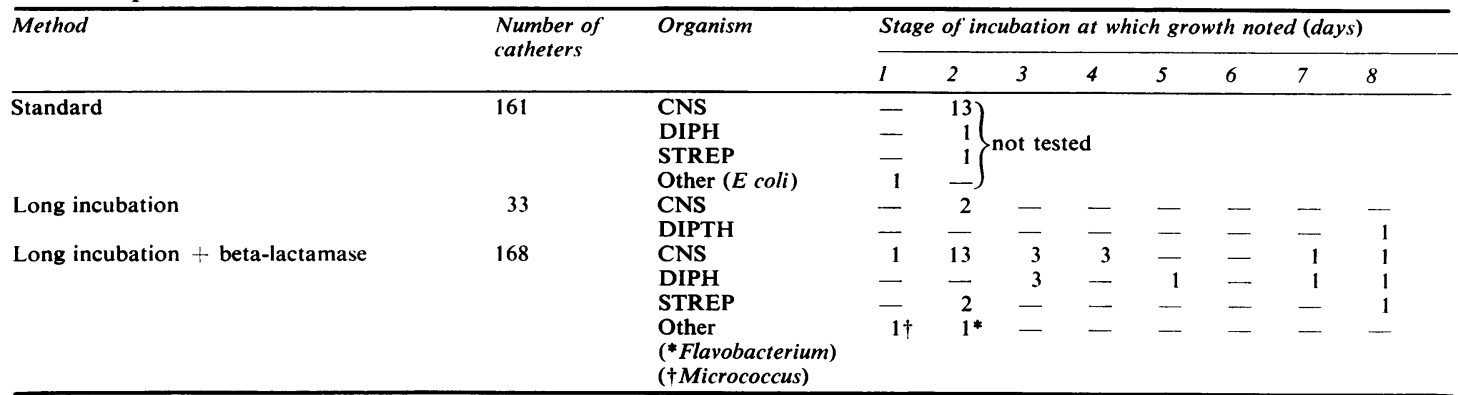

using both prolonged incubation and beta-lactamase is such that the isolation rate within the first two days of incubation would have been 17 isolates from 168 catheter tips, that is $10 \cdot 1 \%$.

\section{Discussion}

It is clear that the use of prolonged incubation (eight days) together with the addition of betalactamase has resulted in a higher isolation rate of bacteria from LA catheter tip cultures. Since the system employed was a "closed" method in order to minimise laboratory contamination the increase is probably caused by either or both alterations to the culture system. Table 1 suggests (but on a small number of specimens) that the mere prolongation of incubation is not responsible for the increase; table 2 reveals that the best results are obtained with the combination of longer incubation and betalactamase addition. However, it is also apparent from table 2 that the increase in isolates above the level obtained by the standard method occurs beyond two days of incubation. Indeed, if the isolation rate is calculated for only the first two days it is not markedly different from that of the standard method $(10.1 \%$ isolation rate in the first two days of the new method compared with $9.9 \%$ for the standard method). Thus, it is likely that the addition of beta-lactamase to neutralise the prophylactic antibiotic results in an increased isolation rate, but the extension of the incubation time beyond two days is necessary for this effect to be fully expressed. This conclusion is further supported by the finding that the increased isolation rate is composed of the summation of increases in coagulase-negative staphylococci, diphtheroids, and streptococci, and not an increased isolation of one particular organism. The range of organisms in which the increases are noted correspond to the spectrum of flucloxacillin.

In summary, therefore, it appears that the addition of beta-lactamase to cultures of left atrial catheter tips will result in the recovery of more organisms, provided that the duration of incubation of the culture allows sufficient time for this effect to be expressed. Obviously, these results will mean that the isolation rate of organisms from catheter tips should be regarded as a commoner event than previously described; in this series it approaches $20 \%$ compared with earlier estimates of $10 \%$. Thus, the possible relevance of this reservoir to early prosthetic valve endocarditis is correspondingly increased. Similarly, the increased incidence of isolations of diphtheroids by this technique would strengthen the case, since both of the organisms most commonly implicated in early prosthetic valve endocarditis can now be recovered from this site.

\section{References}

${ }^{1}$ Freeman R, Hjersing N, Burridge A. Catheter tips cultures on open-heart surgery patients: associations with site of catheter and age of patients. Thorax 1981;36:355-9.

${ }^{2}$ Freeman R, Hjersing N. Bacterial culture of perfusion blood after open-heart surgery. Thorax 1980;35:754-8.

${ }^{3}$ Freeman R, Hjersing N. Species of coagulase-negative staphylococci isolated from catheter tips from openheart surgery patients. Thorax 1980;35:359-63. 